\title{
Le fragile équilibre des intérêts locaux et nationaux dans l'interprétation de la compétence fédérale sur l'aéronautique : l'exemple du parachutisme
}

\section{David Robitaille ${ }^{\star}$}

\section{Introduction}

$\mathrm{Au}$ cour du régime fédéral canadien et de l'interprétation qu'en font les tribunaux, se trouve la recherche d'un équilibre entre les intérêts locaux et les intérêts nationaux. Cette tâche n'est pas aisée, mais est essentielle afin de préserver la capacité des autorités locales à gouverner en fonction des intérêts, des préoccupations et des besoins particuliers de leurs citoyens, tout en permettant l'intervention nécessaire du Parlement sur les enjeux qui concernent le pays dans son ensemble.

La compétence fédérale sur l'aéronautique illustre clairement la complexité à tracer la ligne entre les intérêts locaux et les intérêts nationaux. Les tribunaux canadiens ont de la difficulté à ménager les compétences locales en ce domaine et semblent au contraire considérer l'aéronautique comme une "super " compétence, ne laissant qu'une marge de manœuvre très étroite aux autorités locales en matière d'aménagement durable et sécuritaire du territoire. Or, contrairement à ce que semblent parfois percevoir les tribunaux, ce n'est pas pour mettre arbitrairement des bâtons dans les roues des entreprises ou des activités fédérales que des municipalités refusent parfois que ces dernières s'implantent sur une portion spécifique du territoire ou exigent le respect de normes en matière de gestion du risque. Lobjectif phare étant de répondre aux préoccupations légitimes de citoyens inquiets, telles que le bruit accru, le saccage de milieux humides à grande valeur environnementale, la pollution de l'air qu'ils respirent et de l'eau qu'ils boivent, et du développement inconséquent du territoire ${ }^{1}$.

Les municipalités ne peuvent pas adopter de règlements qui régissent directement ou concernent l'aéronautique par leur caractère véritable, sans quoi ils seraient déclarés inconstitutionnels. Les règlements municipaux d'application générale qui concernent l'aménagement du territoire ainsi que l'environnement, la santé et la sécurité des citoyens peuvent toutefois s'appliquer aux activités aéronautiques. Par contre, ces règlements ne doivent pas entraver le cœur ou les aspects essentiels de cette compétence fédérale, ni être en conflit avec la réglementation fédérale.

Le plus récent arrêt de la Cour d'appel du Québec sur la question, Procureure générale $d u$ Québec c. Leclerc ${ }^{2}$, montre bien la difficulté qu'ont les municipalités à faire respecter leurs compétences en matière d'aéronautique. Cette affaire, dont la Cour suprême a refusé l'autorisation de pourvoi ${ }^{3}$, mettait en cause l'applicabilité constitutionnelle du Règlement sur le zonage et le lotissement ("Règlement sur le zonage ») et du Règlement sur les permis et les certificats ( Règlement sur les permis ») de la Ville de Lévis à légard d'activités commerciales et sportives de parachutisme ayant cours en zone agricole. Seule la doctrine de l'exclusivité des compétences fut soulevée en première instance par la défender- 
esse, Albertine Leclerc, à l'encontre des constats d'infraction qui avaient été émis par la Ville ${ }^{4}$.

Pour les motifs que nous analyserons ci-dessous, la Cour d'appel du Québec en est venue à la conclusion que le Règlement sur les permis s'applique à l'entreprise, mais que le Règlement sur le zonage, qui prohibe notamment les activités de parachutisme et de centre de formation en parachutisme sur une partie du territoire municipal zonée agricole était, quant à lui, inapplicable. Les opérations mêmes de l'aérodrome, qui bénéficiaient de droits acquis, nétaient pas en cause dans cette affaire.

\section{L'applicabilité de principe des règlements municipaux aux activités fédérales : l'exigence d'un permis n'est pas une entrave}

Devant la Cour d’appel, la Procureure générale du Canada ( $P G C$ ») plaidait quaucune loi provinciale ou règlement municipal ne peut s'appliquer à une activité fédérale tant qu'un juge n'a pas d'abord constaté l'absence d'entrave dans un jugement. Autrement dit, la PGC invitait la Cour d'appel à établir une présomption d'inapplicabilité du droit municipal à ces activités. Dans ce scénario, chaque fois qu'une municipalité aurait souhaité que sa réglementation soit respectée par une entreprise menant des activités fédérales, il aurait fallu l'intervention d'un juge. Si la Cour d’appel avait confirmé ce point, l'applicabilité du droit provincial et municipal aux entreprises fédérales aurait été gravement menacée. Cela aurait ouvert la possibilité, pour ces entreprises, à se soustraire beaucoup plus facilement aux obligations imposées par la province et les municipalités. La Cour d'appel n'a pas fait droit à cet argument, réitérant la présomption d'applicabilité, ou l'applicabilité de principe, du droit provincial et municipal aux entreprises fédérales ${ }^{5}$, conformément aux enseignements de la Cour suprême du Canada ${ }^{6}$. La doctrine du caractère véritable demeure donc la première doctrine de droit constitutionnel canadien. Pour être soustraites du droit local valide, les entreprises fédérales ont encore le fardeau de démontrer l'entrave constitutionnelle ${ }^{7}$, malgré l'invitation faite à la Cour d'appel d'inverser ce fardeau.
La Cour d’appel a ainsi jugé applicable le Règlement sur les permis de la municipalité. En effet, l'obligation d'obtenir un permis de construction et de transmettre des informations importantes à la municipalité relativement aux activités aéronautiques ne constitue pas en soi une entrave constitutionnelle ${ }^{8}$. La Cour d'appel a également confirmé qu'il faut présumer de la bonne foi des administrations locales ${ }^{9}$. Celles-ci ne peuvent pas se servir de ces exigences relatives aux permis comme moyens indus pour retarder ou interdire ces activités fédérales ${ }^{10}$. Largument de la PGC selon lequel ce «type de régime exige généralement de l'administré [...] [se conforme] à une panoplie de règles et de normes ${ }^{11}$ a conséquemment été rejeté.

Les règlements municipaux similaires imposant l'obtention de permis et la transmission d'informations utiles aux municipalités devraient donc généralement s'appliquer aux activités faisant lobjet de compétences fédérales. Il s'agit d'une victoire importante pour les municipalités, compte tenu de la jurisprudence minoritaire et encore en émergence dans le domaine, et des tentatives des entreprises et de la PGC pour faire reconnaître comme une potentielle entrave constitutionnelle la simple exigence d'un certificat provincial ou d'un permis municipal ${ }^{12}$. Si les municipalités ne peuvent refuser de délivrer un permis, ce qui équivaudrait à une entrave, elles doivent néanmoins être informées des activités qui se déroulent dans leur propre juridiction, ce qui constitue un élément essentiel de l'exercice de la compétence municipale. Disposer d'une information pertinente et à jour permet aux municipalités de connaître la nature des activités et des produits auxquels elles font face, de répondre plus efficacement aux situations urgentes et de limiter les conséquences de sinistres sur l'environnement, la santé et la sécurité des résidents ${ }^{13}$. Cela est aussi important pour permettre aux municipalités de remplir adéquatement l'une de leurs fonctions principales, soit d'assurer, en tant que gouvernement de proximité, des services publics essentiels et de qualité aux citoyens et entreprises sur leur territoire ${ }^{14}$.

Le jugement de la Cour d'appel dans $P G Q$ $c$ Leclerc vient confirmer que les entreprises 
ou citoyens menant des activités fédérales ou, plus exactement, des activités qui sont en partie régies par une compétence fédérale - ne peuvent généralement le faire sans tenir compte au préalable du cadre réglementaire municipal d'application générale établi et mis en place afin d'assurer l'aménagement harmonieux, cohérent, durable et sécuritaire du territoire, dont les municipalités sont les premières responsables ${ }^{15}$.

\section{Le précédent spécifique ou les circonstances exceptionnelles justifiant l'application de la doctrine de l'exclusivité}

La doctrine de l'exclusivité des compétences permet de protéger le cœur et le contenu essentiel des compétences fédérales contre les entraves de la réglementation provinciale ou municipale. Compte tenu de ses effets draconiens, soit la mise à l'écart de normes provinciales ou municipales validement adoptées, la Cour suprême demande aux tribunaux de n'y avoir recours que dans les "situations déjà traitées dans la jurisprudence ${ }^{16}$. Cela signifie que "l'absence de décisions antérieures préconisant son application à l'objet du litige justifiera en général le tribunal » de ne pas appliquer cette doctrine ${ }^{17}$. Devant la Cour d'appel de l'Alberta, deux interprétations sopposaient sur le sens de ces motifs de la Cour suprême dans l'arrêt de principe Banque canadienne de l'Ouest c Alberta ( « BCO ») ${ }^{18}$. Le PGC et l'entreprise soutenaient pouvoir bénéficier de la doctrine de l'exclusivité dès qu'une compétence fédérale à légard de laquelle la jurisprudence l'a déjà appliquée est en cause dans un litige, et ce indépendamment du contexte particulier de chaque affaire. Si la Cour d'appel de l'Alberta avait entériné cette interprétation, la portée de la doctrine de l'exclusivité aurait été élargie, au détriment des intérêts municipaux. Sur la base de la jurisprudence de la Cour suprême, la municipalité a proposé une interprétation plus étroite, que la Cour d’appel a confirmée. Ainsi, il ne suffit pas que le litige mette en cause une compétence fédérale pour invoquer la doctrine de l'exclusivité. Il importe également que les contextes factuels et juridiques des deux affaires soient suffisamment proches, c'est-à-dire que le cour de la compétence fédérale invoqué ait déjà été protégé par cette doctrine dans une décision antérieure ${ }^{19}$.
Compte tenu de cette interprétation restreinte de la doctrine de l'exclusivité, la protection des compétences locales en aménagement du territoire s'en trouve maintenue. Cette décision va à l'encontre de l'applicabilité élargie que souhaitait lui donner la PGC et de la propension centralisatrice et unilatéraliste des compétences fédérales.

Puisquaucun précédent n'a établi que le parachutisme est absolument essentiel à la navigation aérienne ${ }^{20}$, il nous semble toutefois que la Cour d'appel du Québec aurait dû « se demander s'il est possible de trancher la question constitutionnelle sur un autre fondement $»^{21}$. En effet, il est bien établi que la doctrine de l'exclusivité doit demeurer restreinte, puisqu'elle ne reflète pas les valeurs constitutionnelles dominantes du fédéralisme moderne ${ }^{22}$. Si la Cour suprême n'a pas totalement fermé la porte à cette doctrine en l'absence d'un précédent spécifique, elle décourage néanmoins explicitement son application dans ce contexte. La Cour suprême souligne que " [1]e principe des compartiments étanches fixes sur lequel elle repose va à contre-courant de l'évolution de l'interprétation constitutionnelle canadienne, qui tend vers les notions plus souples du double aspect et du fédéralisme coopératif ${ }^{23}$. La Cour suprême accorde d'ailleurs une importance significative à l'identification au préalable d'un précédent spécifique ${ }^{24}$. Elle affirme que «la jurisprudence constitue un guide utile pour bien cerner le cœur d'un chef de compétence $»^{25}$. À notre connaissance, depuis l'arrêt $B C O$, la Cour suprême n'a d'ailleurs jamais appliqué la doctrine de l'exclusivité à de nouveaux domaines, c'est-àdire à des matières précises qui n’en ont jamais fait l'objet auparavant. Elle a même refusé de le faire à deux reprises ces dernières années ${ }^{26}$.

Puisqu'aucun précédent n’a reconnu le parachutisme comme une activité faisant partie du cœur irréductible de la compétence sur la navigation aérienne ${ }^{27}$, que la doctrine de l'exclusivité a une portée restreinte et constitue " une sorte de 'dernier recours' en droit constitutionnel $»^{28}$, il nous semble que la Cour d'appel du Québec aurait dû, comme en a jugé la Cour d'appel de l'Ontario, expliquer les circonstances exceptionnelles justifiant l'utilisation de cette doctrine en matière de parachutisme ${ }^{29}$. La juge de première 
instance, qui avait eu le bénéfice d'entendre un expert en matière d'aéronautique, nous semblait à bon droit avoir refusé d'appliquer cette doctrine à un nouveau domaine $\mathrm{e}^{30}$.

\section{L'indivisibilité mal comprise de la compétence fédérale sur l'aéronautique}

\section{A. Remise en contexte des arrêts-clés sur l'aéronautique}

Les arrêts COPA, Lacombe, Construction Montcalm, Johannesson et Mississauga ne répondent pas à toutes les questions relativement à l'interaction entre l'aéronautique et les compétences locales. Lactivité en cause dans l'arrêt COPA était l'aviation, que la législation provinciale empêchait via l'interdiction de construire des aérodromes en territoire agricole. La Cour suprême du Canada a refusé de scinder en deux une même matière et conclut que l'aviation locale était indivisible de l'aviation interprovinciale et internationale. Dans PGQ c Leclerc, le parachutisme était aisément distinguable de la compétence plus générale sur l'aéronautique et plus spécifique sur la navigation par avion. Il nétait pas question de séparer en deux la compétence sur le parachutisme. Les motifs de la Cour suprême dans l'arrêt Lacombe reposent, par ailleurs, sur les doctrines du caractère véritable et des pouvoirs accessoires. C'est notamment le cas dans d'autres décisions sur lesquelles s'appuyait l'entreprise et la PGC dans la saga du parachutisme ${ }^{31}$. La Cour suprême concluait dans Lacombe que le règlement municipal contesté avait été adopté, à la base, pour interdire les voyages commerciaux d'hydravions sur le lac Gobeil ${ }^{32}$. Le règlement avait donc été déclaré invalide, puisqu'il ne se rattachait pas à la compétence provinciale sur l'aménagement du territoire ${ }^{33}$. Les motifs très courts de la Cour suprême sur la doctrine de l'exclusivité constituaient, en pratique, un obiter dictum ${ }^{34}$. La Cour suprême a rendu l'arrêt Johannesson dans un contexte similaire. La Municipal Act du Manitoba autorisait expressément les municipalités à réglementer l'aviation en prévoyant à son article 921 que « [a]ny municipal corporation may pass by-laws for licensing, regulating, and, within certain defined areas, preventing the erection, maintenance and continuance of aerodromes or places where aeroplanes are kept for hire or gain $»^{35}$. Le règlement fût invalidé en raison de son caractère véritable ${ }^{36}$. La doctrine moderne de l'exclusivité des compétences nétait pas en cause dans cette affaire, telle qu'interprétée depuis l'arrêt $B C O$ dans le contexte du fédéralisme coopératif ${ }^{37}$, du principe de l'interprétation coordonnée $^{38}$ et du principe de subsidiaritée ${ }^{39}$. Dans les arrêts Construction Montcalm et Murray Hill Limousine, la doctrine de l'exclusivité nétait pas directement en cause puisque la Cour suprême et la Cour d'appel du Québec y jugeaient, respectivement, que les entreprises ou activités concernées étaient de nature locale et provinciale ${ }^{40}$, et non fédérale. Enfin, l'arrêt Mississauga dans lequel la Cour d'appel de l'Ontario jugeait inapplicable l'agrandissement de l'aéroport Pearson en vertu du Code du bâtiment de l'Ontario a été rendu en 2001 , soit bien avant l'arrêt $B C O^{41}$, et le resserrement des critères de la doctrine de l'exclusivitét2. La solidité de cet arrêt comme précédent a d'ailleurs été mise en doute par la Cour supérieure de l'Ontario ${ }^{43}$, tandis que la Cour suprême affirmait que les jugements rendus avant le changement de paradigme de 2007 dans $B C O$ ont « peu de valeur jurisprudentielle $»^{44}$.

\section{B. L'indivisibilité du cœur de la compétence fédérale ne signifie pas que toute la compétence soit indivisible}

La Cour d'appel du Québec dans PGC c Leclerc a souligné, dans des motifs plutôt succincts et en se basant sur ce qui nous paraît constituer des considérations plutôt générales, en quoi le parachutisme fait partie du cœur de la compétence fédérale sur l'aéronautique :

Pour le juge de la Cour supérieure, le parachutisme est, par définition, une activité aéronautique et il est impossible de dissocier les activités aéronautiques des aérodromes où elles se déroulent.

J'abonde dans le sens du juge de la Cour supérieure. À mon avis, le parachutisme n'est pas un élément accessoire à l'exploitation de l'aérodrome ou des aéronefs. Le parachutisme est certainement une activité qui se pratique dans l'espace aérien et qui est indissociable « de la navigation aérienne prise comme un tout $»$. Il est donc nécessaire que cette activité fasse 
partie du cour de la compétence en matière d'aéronautique pour permettre au fédéral de réaliser les objectifs d'efficacité et de sécurité pour lesquels cette compétence lui a été attribuée.

Dans COPA, la juge McLachlin conclut qu'il est établi depuis longtemps que "l'endroit où un avion peut décoller et se poser est une matière que protège la doctrine de l'exclusivité des compétences ». Par analogie, j’estime que l'endroit où l'on peut pratiquer le parachutisme est un élément du contenu essentiel de la compétence fédérale en matière d’aéronautique [nos soulignements; références omises] ${ }^{45}$.

Si le parachutisme fait certainement partie de la compétence fédérale sur l'aéronautique et qu'il se pratique, évidemment, dans les airs, il ne s'ensuit pas qu'il fasse automatiquement partie du cœur de cette compétence ${ }^{46}$. Avec égards, la Cour d'appel fait sur ce point une interprétation extensive de la notion d'indissociabilité, ou d'indivisibilité, que la Cour suprême applique dans un tout autre contexte dans l'arrêt COPA. Dans cette affaire, la Cour suprême faisait notamment référence à l'indivisibilité de la compétence fédérale en raison de la distinction avancée par un intervenant entre l'aviation locale et l'aviation interprovinciale et internationale. La Cour suprême a souligné dans cet arrêt que les étapes du décollage, du vol d'avion et de l'atterrissage sont indissociables et forment un tout.

Le Procureur général de la Colombie-Britannique proposait à la Cour suprême d'attribuer à la province, plutôt qu'au Parlement, la compétence exclusive sur l'aviation locale ${ }^{47}$. La Cour suprême a jugé qu'il était impossible de distinguer l'aviation locale de l'aviation interprovinciale compte tenu de la nature de cette activité :

[...] les aspects locaux de l'aviation relèvent de la compétence fédérale parce que la matière visée par la navigation aérienne est «indivisible». Utilisant defaçoninterchangeable les mots " aéroport " et " aérodrome ", il a conclu que [TRADUCTION] « tout comme il est impossible d'établir une distinction entre les vols intraprovinciaux et interprovinciaux, l'emplacement et la réglementation des aéroports ne peuvent être associés ni à l'un ni à l'autre, ou dissociés de la navigation aérienne prise comme un tout »(p. 314). Ce point de vue reflète le fait que les aéroports et les aérodromes canadiens forment un réseau de lieux d'atterrissage qui, ensemble, facilitent le transport aérien et assurent la sécurité.

Il est donc clair que la compétence fédérale en matière d'aéronautique englobe le pouvoir de déterminer l'emplacement des aérodromes. La question suivante est de savoir si ce pouvoir se trouve protégé au cœur d'un pouvoir fédéral [notre italique]. ${ }^{48}$

C'est donc précisément dans le contexte du transport par avion et de la détermination de l'emplacement d'un aérodrome que la Cour suprême jugeait inapplicable la loi provinciale :

Le transport aérien est un élément indispensable de la vie moderne. Alors même que notre dépendance à l'égard de l'avion s'est accrue, les demandes émanant du domaine de l'aviation entrent pourtant de plus en plus en conflit avec d'autres intérêts. Les avions doivent décoller et atterrir. Ils ont donc besoin de terres ou d'eau. Tout terrain ou plan d'eau utilisé pour l'aviation ne peut être utilisé à d'autres fins. La question en l'espèce et dans le pourvoi connexe, Québec (Procureur général) c. Lacombe, 2010 CSC 38, [2010] 2 R.C.S. 453 , est de savoir quel ordre de gouvernement a le dernier mot sur l'emplacement des terrains d'aviation et des aérodromes [nos soulignements $]^{49}$.

Or, dans PGQ c Leclerc, les opérations mêmes de l'aérodrome nétaient pas visées par la municipalité. Contrairement à ce que plaidait le Procureur général de la Colombie-Britannique dans COPA, personne ne demandait à la Cour d'attribuer la compétence sur le parachutisme exclusivement aux provinces ou aux municipalités. En effet, il ne s'agissait pas d'essayer de scinder en deux la compétence législative sur un même sujet pour en attribuer une partie aux provinces et aux municipalités comme le soutenait un intervenant provincial dans COPA, ni de distinguer les aspects locaux et les aspects nationaux d'une même activité. Il s'agissait, selon nous, strictement d'un cas d'applicabilité de règlements locaux valides, adoptés en vue d'assurer la santé et la sécurité des citoyens et le développe- 
ment structuré du territoire, à une activité accessoire à l'aviation et à l'aéronautique.

Toutes les activités liées à l'aéronautique, ou faisant partie de cette compétence fédérale, ne font pas partie du cœur de cette compétence ${ }^{50}$. Le litige portait sur une activité sportive dont la dimension locale ne fait, à notre avis, aucun doute. En matière de parachutisme, nous sommes loin des considérations d'intérêt national qui ont, avec raison, justifié nos tribunaux d'attribuer au Parlement la compétence sur l'aviation au fédéral. Tel quécrivait le juge Locke dans l'arrêt Johannesson :

There is a very large passenger traffic between the provinces and to and from foreign countries, and a very considerable volume of freight traffic not only between the settled portions of the country but between those areas and the northern part of Canada, and planes are extensively used in the carriage of mails. That this traffic will increase greatly in volume and extent is undoubted. While the largest activity in the carrying of passengers and mails east and west is in the hands of a government controlled company, private companies carry on large operations, particularly between the settled parts of the country and the North and mails are carried by some of these lines. The maintenance and extension of this traffic, particularly to the North, is essential to the opening up of the country and the development of the resources of the nation. It requires merely a statement of these well recognized facts to demonstrate that the field of aeronautics is one which concerns the country as a whole. ${ }^{51}$

Plusieurs juges de la Cour suprême l'ont souligné, c'est également dans le contexte de la ratification par le Canada de la Convention relative à l'aviation civile internationale (la "Convention ») de 1944, et des obligations s'y afférentes, que le Canada avait la responsabilité de transposer en droit canadien, qu'a été attribué au Parlement la compétence sur l'aéronautique ${ }^{52}$. Or, il nous semble révélateur que cette Convention ne fasse aucunement mention du parachutisme. Il en ressort en outre qu'elle ne considère pas le parachute comme un aéronef et ne porte que sur le transport par avion de passagers, de marchandises et de courrier ${ }^{53}$. Ce qui importe, et ce qui a conséquemment motivé l'adoption de la Loi sur l'aéronautique, comme le soulignait la Cour suprême dans l'arrêt Johannesson ${ }^{54}$, ce sont les engagements internationaux que le Canada prenait alors vis-à-vis ses cosignataires en ce qui concerne l'aviation civile et commerciale. Avec respect, les tribunaux d'aujourd'hui ont parfois tendance à oublier ce contexte particulier au profit d'interprétations impressionnistes, larges et libérales, voire presque " mystiques", de l'aéronautique et des autres compétences fédérales sur les transports.

Il nous semble d'ailleurs évocateur de constater que larticle 29 de la Convention impose l'obligation pour un aéronef d'avoir à bord son certificat d'immatriculation, alors que le Parlement exempt, dans sa loi, les parachutes de l'obligation faite aux avions dêtre immatriculés ${ }^{55}$. C'est aussi ce que relevait la juge de première instance dans cette affaire, sur la base du témoignage de l'expert, avant de conclure que le parachutisme ne fait pas partie du cour de la compétence fédérale : " Autre distinction. [...] [I]l n'est pas requis d'immatriculer un parachute ou un deltaplane, spécifie M. Eralp. Il s'agit pourtant d'une exigence pour les avions qui eux ont le pouvoir de se déplacer, dans l'espace aérien, d'une province à l'autre $»^{56}$. Le Parlement n'a pas non plus rendu nécessaire pour le propriétaire d'un aéronef de détenir une assurance-responsabilité pour les passagers faisant du parachute, contrairement à l'obligation imposée à tout transporteur aérien de passagers ${ }^{57}$. Le Parlement semble donc lui-même considérer le parachutisme comme un domaine incident ou distinct de l'aviation. Or, si le Parlement ne peut unilatéralement élargir la portée de ses compétences par l'adoption d'une loi, celle-ci peut, en revanche, démontrer qu'il considère une activité comme étant moins essentielle à sa compétence ${ }^{58}$. En outre, la Cour d'appel de l'Alberta, dans une affaire où le PGC nétait toutefois pas impliqué, suggérait, compte tenu des admissions des parties, que le parachutisme est une activité "incidente " à l'aviation [notre traduction $]^{59}$.

Malgré ce qui précède, la Cour d'appel du Québec n'explique pourtant que sommairement 
pourquoi le parachutisme serait absolument nécessaire à l'aviation. Son explication se limite essentiellement à établir, par une analogie discutable avec le décollage et l'atterrissage d'avions, que l'endroit où l'on peut pratiquer le parachutisme fait partie du cœur de la compétence fédérale sur l'aéronautique, et en est donc indissociable ${ }^{60}$. Avec égards, cela ne peut être qualifié de ce que la Cour d'appel de l'Ontario qualifiait $\mathrm{d}^{\prime}$ " exceptional rationale $»^{61}$, permettant d'appliquer la doctrine de l'exclusivité en dehors d'un précédent spécifique.

L'honorable Gascon soulignait à juste titre, lorsqu'il siégeait à la Cour supérieure du Québec, à propos de la détermination du cœur d'une compétence fédérale, que "[p]our être indispensable, il faut que la spécificité fédérale de la compétence ne puisse exister sans lélément en question " [nos soulignements $]^{62}$. Il nous semble évident que la navigation aérienne et l'aéronautique peuvent aisément exister sans l'activité de parachutisme. La Cour d'appel du Québec nous semble respectueusement avoir erronément appliqué le raisonnement inverse, selon lequel l'espace aérien et un avion sont nécessaires au parachutisme, plutôt que de se demander $a$ contrario si la navigation aérienne peut exister sans parachute. Il est bien connu, et peut-être même de connaissance d'office, que dans la vaste majorité des avions, il n'y a aucun parachute. On ne peut assimiler un parachute à une piste d'atterrissage, aux normes de construction des avions ou des aéroports, à l'exploitation sécuritaire d'un avion, ou à l'aire d'attente des passagers, notamment que la jurisprudence a reconnu comme faisant partie du cœur de la compétence fédérale. Compte tenu, par ailleurs, de l'absence de répercussions sur l'uniformité du droit canadien en la matière, la Cour d'appel du Québec aurait dû conclure, avec respect, que le parachutisme n'est pas une activité sportive sans laquelle l'aviation ou l'aéronautique ne pourrait absolument pas fonctionner. La Cour suprême soulignait, en effet, que l'absence d'impact important sur l'uniformité du droit fédéral des transports en l'espèce, la navigation - une compétence fédérale vaste, justifie de ne pas appliquer la doctrine de l'exclusivité63. Contrairement à l'aviation, elle-même susceptible de franchir les frontières provinciales, le parachutisme est une activité qui se déroule normalement sur un territoire relativement bien circonscrit et déterminé. Nous soumettons avec respect que la comparaison faite par la Cour d'appel du Québec est erronée et mène à un élargissement indu de la compétence fédérale en assimilant le cœur de la compétence à l'entièreté de la compétence. Si le parachute fait partie des éléments irréductibles de l'aéronautique, nous voyons mal ce qui n'en fera pas partie. Enfin, dans la mesure où la Cour supérieure du Québec reconnaissait que le parachutisme " n'est peut-être pas toujours nécessaire à l'aéronef qu'est l'avion $"{ }^{64}$, elle aurait logiquement dû conclure, selon notre compréhension de la jurisprudence, que cette activité ne fait pas partie du cour de la compétence fédérale.

En « abond[ant] dans le sens du juge de la Cour supérieure ${ }^{65}$ sur ce point, la Cour d'appel du Québec avalise une méthode douteuse, voire inconstitutionnelle, de détermination du cœur d'une compétence fédérale. En effet, la Cour supérieure du Québec s'est largement, sinon exclusivement, fondée sur la définition du mot « aéronef » prévue à l'article 3(1) de la Loi sur l'aéronautique ${ }^{66}$, une loi fédérale, pour déterminer si le parachutisme fait partie des éléments essentiels de la compétence fédérale ${ }^{67}$. Ce faisant, avec égards, il semble avoir incidemment été reconnu au Parlement le pouvoir de déterminer unilatéralement l'étendue de ses compétences, une tâche qui revient pourtant normalement aux tribunaux dans un partage constitutionnel des compétences ${ }^{68}$.

\section{Conclusion}

Lorsqu'il est question de la compétence locale sur l'aménagement du territoire et du zonage, d'une part, et de la compétence fédérale sur l'aéronautique, d'autre part, le réflexe quasi automatique de plusieurs juristes est de considérer intouchable l'activité ou l'entreprise fédérale exerçant en ce domaine. Pourtant, l'état de la jurisprudence ne devrait plus permettre ce genre de réflexe. Le droit constitutionnel, avec ses doctrines du caractère véritable et du double aspect, de l'exclusivité et de la prépondérance qui distinguent la validité, l'applicabilité et 
l'opérabilité des normes locales, est plus complexe que cela. Le droit constitutionnel n'autorise pas les raisonnements expéditifs, lesquels profitent d'ailleurs le plus souvent, il nous semble, au Parlement. Dans un litige où l'aéronautique fédérale, l'aménagement, ou le zonage local du territoire entrent en tension, le réflexe est souvent de penser que la jurisprudence règle déjà toute question.

La Cour supérieure et la Cour d'appel du Québec dans $P G Q$ c Leclerc nous semblent avoir eu ce même réflexe, et ont respectueusement omis d'afficher la prudence nécessaire au maintien de l'équilibre des compétences provinciales et fédérales afin de protéger un élément accessoire à l'aviation. En privilégiant une interprétation extensive du concept de contenu minimum et irréductible ou de noyau de la compétence fédérale, le jugement de la Cour d'appel du Québec a ainsi pour effet d'élargir le champ d'application de la doctrine de l'exclusivité. Sur la base d'une interprétation large et décontextualisée de la jurisprudence antérieure, cela risque également de contribuer à l'établissement de nouvelles enclaves autour d'entreprises ou de compétences fédérales ${ }^{69}$.

Il est temps que les tribunaux, au premier chef la Cour suprême du Canada, fassent une lecture plus harmonieuse des compétences locales, notamment en matière d'aménagement du territoire, d'environnement, de la santé, de la sécurité et de l'urbanisme, en relation avec la compétence fédérale sur l'aéronautique. L'unilatéralisme prévaut encore trop souvent dans d'autres domaines de compétence fédérale tels que les transports fédéraux et les télécommunications Ainsi, il faut reconnaître aux gouvernements locaux et à leurs citoyens le pouvoir de déterminer des portions raisonnables du territoire à protéger contre la construction d'infrastructures fédérales de transport ou de communications. Ce pouvoir doit être conditionnel au maintien de l'efficacité de ces compétences, et ne devrait pas mettre en échec l'uniformité du droit canadien ni l'intérêt national d'avoir un système de transport et de communications effectif ${ }^{70}$. Si les compétences sont « coordonnées, et non subordonnées $»^{71}$, et que les tribunaux se soucient réellement des préoccupations des citoyens sur le territoire local $^{72}$, ils devraient emprunter cette seule voie.

\section{Endnotes}

* Professeur titulaire, Faculté de droit de l'Université d'Ottawa; Avocat-conseil, DHC Avocats, Montréal. Ce texte a bénéficié du soutien financier du Conseil de recherches en sciences humaines du Canada, que nous remercions vivement, dans le cadre du projet "Citoyenneté locale, enjeux environnementaux et partage des compétences : combler le déficit démocratique issu d'un fédéralisme bi-dimensionnel ", que le soussigné mène avec ses collègues Benoît Frate et Lucie Lamarche, respectivement du Département d'études urbaines et touristiques et du Département des sciences juridiques de l'Université du Québec à Montréal. Le soussigné a été avocat-conseil auprès de la municipalité dans les affaires Procureure générale du Québec c Leclerc, 2018 QCCA 1567 et Ville de Lévis c Albertine Leclerc, et al (demande d'autorisation de pourvoi refusée, CSC, 23 mai 2019, dossier 38414) analysées dans ce texte.

1 Voir, notamment, Alie Dostie, «Boeing 737-200 à Saint-Hubert: des citoyens expriment leurs inquiétudes à la Ville ", Le Reflet, (21 mars 2019), en ligne: le Reflet <www.lereflet.qc.ca/boeing-737 -200-a-saint-hubert-des-citoyens-exprimentleurs-inquietudes-a-la-ville/>; Guy Veillette, " Des citoyens dénoncent une hausse du vol d'hydravions ", Le Nouvelliste, (16 octobre 2017), en ligne: le Nouvelliste <www.lenouvelliste.ca/ actualites/des-citoyens-denoncent-une-haussedu-nombre-de-vols-dhydravions-b7e9a4914a8 3a0ba2bf8a06edaea8ac2>; Radio-Canada, "Les tensions persistent à Saint-Cuthbert au sujet d'un projet d'aérodrome ", Ici Mauricie-Centre$d u$-Québec, (21 novembre 2016), en ligne: CBC Radio-Canada <ici.radio-canada.ca/nouvelle/ 1001398/assemblee-nationale-quebec-bruitavions-contestation-maire-municipalite>; Claire Clancy, " Parkland County residents launch petition to shut down small airport ", Edmonton Journal, (7 octobre 2016), en ligne: CBC Radio-Canada <edmontonjournal.com/ news/local-news/parkland-county-residentslaunch-petition-to-shut-down-small-airport> ; Neil Corbett, "Residents oppose Pitt Meadows runway expansion », Maple Ridge-Pitt Meadows 
News, (24 novembre 2015), en ligne: Maple RidgePitt Meadows News <www.mapleridgenews.com/ news/residents-oppose-pitt-meadows-runwayexpansion/>.

2 Procureure générale du Québec c Leclerc, 2018 QCCA 1567 [PGQ c Leclerc (Cour d'appel)].

3 Ville de Lévis c Albertine Leclerc, et al., CSC, 23 mai 2019, dossier 38414.

4 C'est la raison pour laquelle ce texte ne concerne que la doctrine de l'exclusivité et non la doctrine de la prépondérance fédérale.

5 PGQ c Leclerc (Cour d'appel), supra note 2 aux para 71,76 et 80.

6 Clark c Compagnie des chemins de fer nationaux $d u$ Canada, [1988] 2 RCS 680 aux para 46, 51 et 54. Pour de plus amples développements, voir David Robitaille, " Environnement, aménagement du territoire et transports fédéraux : fédéralisme coordonné ou subordonné ? ", (2018) 48 Revue générale de droit 7 aux pp 36 à 39; David Robitaille, "Le transport interprovincial sur le territoire local : vers un nécessaire équilibre ", (2015) 20 Revue détudes constitutionnelles 76; Martin Z. Olszynski, « Testing the Jurisdictional Waters: The Provincial Regulation of Interprovincial Pipelines ", (2018) 23 Review of Constitutional Studies 91 aux pp 100101,105 et 110 .

7 Ibid.

8 PGQ c Leclerc (Cour d'appel), supra note 2 aux para 79 à 82 .

9 Ibid au para 81. Voir également Procureure générale du Québec c 9105425 Canada Association, 2017 QCCA 426 aux para 65, 76 et 77.

10 Sur ce point, voir les motifs de décision de l'ordonnance MO-057-2017 de l'Office national de lénergie datée du 6 décembre 2017, dans le dossier qui opposait la ville de Burnaby et l’oléoduc Trans Mountain.

$11 P G Q$ c Leclerc (Cour d'appel), supra note 2 au para 80. A contrario, dans le contexte d'un code provincial du bâtiment, voir : Oshawa (City) v 536813 Ontario Limited, 2016 ONCJ 287 aux para 106 à 109.

12 Voir notamment Ville de Mascouche c 9105425 Canada Association, 2018 QCCS 550 aux para 173 et 181-190; Procureure générale du Québec c 9105425 Canada Association, supra note 9 aux para 76-77; Coastal First Nations v British Columbia (Environment), 2016 BCSC 34 aux para 58 et 65; Oshawa (City) v 536813 Ontario Limited, ibid; Leclerc c Lévis (Ville de), 2016 QCCS 6328 aux para 156-157 [Leclerc c Lévis (Cour supérieure)]; Québec (Procureure générale) c IMTT-Québec inc.,
2016 QCCS 4337; Neuville (Ville de) c 9247-9104 Québec inc., 2016 QCCS 113.

13 Voir notamment TransCanada Pipelines Ltd. c Ontario (Ministry of Community Safety and Correctional Services), [2007] OJ No 3014 (ON CS) (QL) aux para 30 et 35, dans laquelle la Cour suprême de l'Ontario a jugé le Code [provincial] de prévention des incendies applicable à Trans Canada.

14 Catalyst Paper Corp. $c$ North Cowichan (District), [2012] 1 RCS 5; 114957 Canada Ltée (Spraytech, Société d'arrosage) c Hudson (Ville), [2001] 2 RCS 241; Produits Shell Canada Ltée c Vancouver (Ville), [1994] 1 RCS 231.

15 TransCanada Pipelines Ltd. c Ontario (Ministry of Community Safety and Correctional Services), supra note 13 aux para 30 et 35 . Voir aussi Burlington Airpark Inc. $v$ Burlington (City), 2014 ONCA 468.

16 Banque canadienne de l'Ouest c Alberta, [2007] 2 RCS 3 au para 77 [BCO].

17 Ibid au para 78.

18 Supra note 16.

19 PQG c Leclerc (Cour d'appel), supra note 2 aux para 51 et 56.

20 Ibid au para 56.

21 Canada (Procureur général) c PHS Community Services Society, [2011] 3 RCS 134 au para 65 [PHS Community Services Society].

22 Rogers Communications Inc. $c$ Châteauguay (Ville), [2016] 1 RCS 467 aux para 62 à 65 [Rogers c Châteauguay]; Québec (Procureur général) c Canadian Owners and Pilots Association, [2010] 2 RCS 536 au para 36 [COPA]; Marine Services International Ltd c Ryan (Succession), [2013] 3 RCS 53 au para 49 [Ryan (Succession)]; BCO, supra note 16 aux para 77-78; Taseko Mines Limited $v$ Canada (Environment), 2017 FC 1100 au para 160.

23 PHS Community Services Society, supra note 21 au para 70. Pour des développements plus approfondis sur le principe du fédéralisme, notamment le fédéralisme coopératif, dans la jurisprudence de la Cour suprême, voir notamment : Eric M. Adams, " Judging the Limits of Cooperative Federalism ", (2016) 76 S.C.L.R. (2 édition) 27; Eugénie Brouillet, La négation de la nation : Lidentité culturelle québécoise et le fédéralisme canadien, Cahiers des Amériques, Septentrion, Québec, 2005; Hugo Cyr, " Autonomy, Subsidiarity, Solidarity: Foundation of Cooperative Federalism », (2014) 23:4 Constitutional Forum 20; Jean-François Gaudreault-Desbiens et Fabien Gélinas, Le fédéralisme dans tous des états : Gouvernance, identité et méthodologie, Bruylant/ Yvon Blais, Bruxelles/Montréal, 2005; Kate Glover, 
«Structural Cooperative Federalism », (2016) 76 S.C.L.R. (2 édition) 45; Warren J. Newman, « The Promise and Limits of Cooperative Federalism as a Constitutional Principle ", (2016) 76 S.C.L.R. ( $2^{\mathrm{e}}$ édition) 67; Jean Leclair, "The Supreme Court of Canada's Understanding of Federalism: Efficiency at the Expense of Diversity ", (2002-2003) 28 Queen's LJ 411; Bruce Ryder, «Equal Autonomy in Canadian Federalism: the Continuing Search for Balance in the Interpretation of the Division of Powers ", (2011) 54 S.C.L.R. (2 édition) 565; Wade K. Wright, "Federalism(s) in the Supreme Court of Canada During the McLachlin Years ", (2018) 86 S.C.L.R. (2 édition) 213; Wade K. Wright, " Courts as Facilitators of Intergovernmental Dialogue: Cooperative Federalism and Judicial Review ", (2016) 72 S.C.L.R. (2 édition) 365.

24 Rogers $c$ Châteauguay, supra note 22 aux para 61 à 64.

25 Ryan (Succession), supra note 22 au para 55; COPA, supra note 22 au para 36.

26 Carter c Canada (Procureur général), [2015] 1 RCS 331 et PHS Community Services Society, supra note 21.

27 PGQ c Leclerc (Cour d'appel), supra note 2 au para 56.

28 Taseko Mines Limited v Canada (Environment), supra note 22 au para 160.

29 Canada Post Corporation v Hamilton (City), 2016 ONCA 767 aux para 94 à 96.

30 Lévis (Ville de) c Leclerc, 2015 CanLII 62548 (QC CM) aux para 165 et 167 [Lévis c Leclerc (Cour municipale)].

31 Québec (Procureur général) c Lacombe, [2010] 2 RCS 453 aux para 30 et 58 [Lacombe]. Voir aussi Chalets St-Adolphe inc. c St-Adolphe d'Howard (Municipalité de), 2011 QCCA 1491 aux para 41et 42, et Québec (Procureure générale) c Larochelle, 2003 CanLII 35051 (QC CA).

32 Lacombe, ibid aux para 20 à 23.

33 COPA, supra note 22 aux para 30 , et 47 à 58 .

34 Ibid au para 66.

35 Johannesson v Municipality of West St. Paul, [1952] 1 SCR 292 à la p. 304 [nous soulignons].

$36 \mathrm{Ibid}$ aux pp 309, 314, 320 et 328.

37 Orphan Well Association c Grant Thornton Ltd., 2019 CSC 5 aux para 66, 105 et 111; BCO, supra note 16 aux para 22 et 24 .

38 Renvoi relatif à la Loi sur les valeurs mobilières, [2011] 3 RCS 837 au para 71.

$39 B C O$, supra note 16 au para 45.

40 Construction Montcalm Inc. c Commission $d u$ salaire minimum du Québec, [1979] 1 RCS 754 aux pp 775 et 776; Murray Hill Limousine Service Ltd. c Batson, 1965 CanLII 657 (QC CQ). Dans cette dernière affaire, la Cour d'appel ne se demandait pas, par ailleurs, si les activités de l'entreprise étaient au cœur de la compétence fédérale, mais si les activités régulières de cette dernières faisaient d'elle un maillon essentiel de l'entreprise fédérale du transport aérien, afin de déterminer si ses relations de travail sont sujettes à la loi fédérale ou à la loi provinciale. La nature locale ou fédérale d'une entreprise, en fonction de ses activités quotidiennes, et la détermination du cœur d'une compétence fédérale, sont deux concepts juridiques complètement distincts.

41 City of Mississauga c Greater Toronto Airports, [2000] OJ No 4086 (CA ON).

42 Ibid au para 41, la Cour d'appel applique l'ancien critère "affects».

43 Oshawa (City) v 536813 Ontario Limited, supra note 11 au para 84; 2241960 Ontario Inc c Scugog (Township), 2011 ONSC 2337 au para 45.

44 Nation Tsilhqot'in c ColombieBritannique, [2014] 2 RCS 256 au para 150; Ryan (Succession), supra note 22 au para 64. Voir également Lacombe, supra note 31 au para 155 (j. Deschamps).

45 PGQ c Leclerc (Cour d'appel), supra note 2 aux para 64 à 66.

46 La Cour suprême a d'ailleurs établi que tous les aspects de la compétence sur l'aéronautique ne relèvent pas du cœur de celle-ci : COPA, supra note 22 aux para 34 à 40; Air Canada c Ontario (Régie des alcools), [1997] 2 RCS 581.

47 COPA, supra note 22 au para 32.

48 Ibid aux para 33 et 34 . Pour des développements plus approfondis sur les arrêts COPA et Lacombe, voir : Robin Elliot, "Quebec (Attorney General) v Lacombe; et Quebec (Attorney General) v C.O.P.A.: Ancillary Powers, Interjurisdictional Immunity and 'The Local Interest Land Use Planning against the National Interest in a Unified System of Aviation Navigation" ", (2011) 55 S.C.L.R. (2 édition) 403 (QL).

49 COPA, supra note 22 au para 1.

50 Ibid aux para 34 à 41.

51 Johannesson, supra note 35 aux pp 326-327. Dans ce contexte, certains ont mis en doute le fait que les petits aérodromes privés, contrairement aux aéroports commerciaux, soient véritablement d'intérêt national : Elliot, supra note 48 au para 58; Ryder, supra note 23 aux pp 590 à 592.

52 Johannesson, supra note 35, aux pp 303 (juge en chef Rinfret), 307-308 (j. Kerwin), et 310-311 (j. Kellock), ce dernier citant avec approbation le Vicomte Dunedin dans l'Avis sur les radiocommunications, [1932] A.C. 304. 
53 Convention relative à l'aviation civile internationale, signée à Chicago, 1944, Organisation de l'aviation civile internationale, Nations Unies, en ligne : $<$ https://www.icao.int/about-icao/Pages/default. aspx $\geq$, notamment les articles 22, 29 et 96.

54 Johannesson, supra note 35 aux pp 303, 307-308, et 310-311.

55 Norme 623 - Normes d'opérations aériennes spécialisées, Règlement de l'aviation aérienne, DORS/96-433, art 202.13.

56 Lévis (Ville de) c Leclerc (Cour municipale), supra note 30 au para 164 .

57 Règlement de l'aviation aérienne, supra note 55, art 606.02(2), (3) et (4).

58 BCO, supra note 16 au para 91.

59 Mercier v Alberta (Attorney General), 1997 ABCA 161 au para 4.

60 PGQ c Leclerc (Cour d'appel), supra note 2 aux para 66 et 67.

61 Canada Post Corporation v Hamilton (City), supra note 29 au para 96.

62 Marcotte c Banque de Montréal, 2009 QCCS 2764 au para 615.

63 Ryan (Succession), supra note 22 au para 64.

64 Leclerc c Lévis (Cour supérieure), supra note 12 au para 141.

65 PGQ c Leclerc (Cour d'appel), supra note 2 au para 65.
66 Leclerc c Lévis (Cour supérieure), supra note 12 au para 129.

67 Ibid aux para 121 à 146 , et plus particulièrement aux para 131, 134, 142, 143, 145 et 146.

$68 B C O$, supra note 16 au para 4. Voir également 9171-7702 Québec Inc. v Canada, 2013 FC 832 au para 24; Daniels c Canada, 2013 CF 6 (CanLII) aux para 112 et 113, conclusion infirmée en partie en appel dans l'affaire Canada (Affaires indiennes) $c$ Daniels, 2014 CAF 101 (CanLII) au para 159, mais rétablie en Cour suprême dans l'arrêt Daniels $c$ Canada (Affaires indiennes et du Nord canadien), [2016] 1 RCS 99, 2016 CSC 12 (CanLII) aux para 57 et 58.

69 PHS Community Services Society, supra note 21 au para 64; Colombie-Britannique (Procureur général) c Lafarge Canada Inc., [2007] 2 RCS 86 au para 72; Clark c Compagnie des chemins de fer nationaux du Canada, supra note 6 aux para 46, 51 et 54 .

70 Elliot, supra note 48 au para 58.

71 Renvoi relatif à la Loi sur les valeurs mobilières, supra note 38 au para 71.

72 Tel que souligné dans Ryder, supra note 23 aux pp 590 à 592, les motifs majoritaires dans les arrêts COPA et Lacombe ne témoignent pas d'une grande sensibilité à l'importance des intérêts locaux. 
